\title{
Atividades diárias de Tropidurus hispidus (Squamata: Iguania: Tropiduridae) na Ilha do Monte Cristo, Baía de Todos-os-Santos, Bahia, Brasil
}

\author{
Danilo Sabino da Silva Lima*1, Rodrigo Santos Siqueira², Eduardo José dos Reis Dias \& Rejâne M. \\ Lira-da-Silva ${ }^{4}$
}

${ }^{1}$ Núcleo de Etologia e Evolução, Departamento de Zoologia, Instituto de Biologia, Universidade Federal da Bahia, Salvador, Bahia, Brasil. E-mail: *danilosabino240@hotmail.com.

2Laboratório de Biologia e Ecologia de Vertebrados (LABEV), Pós-Graduação em Diversidade Animal, Instituto de Biologia, Universidade Federal da Bahia, Salvador, Bahia, Brasil. E-mail: r.rodrigosiqueira@gmail.com.

${ }^{3}$ Laboratório de Biologia e Ecologia de Vertebrados (LABEV), Departamento de Biociências, Campus Alberto de Carvalho, Universidade Federal de Sergipe, Itabaiana, Sergipe, Brasil.E-mail: ejrdias@hotmail.com.

${ }^{4}$ Núcleo de Ofiologia e Animais Peçonhentos da Bahia (NOAP/UFBA), Instituto de Biologia, Universidade Federal da Bahia, Salvador, Bahia, Brasil.E-mail: rejane@ufba.br.

Abstract. Daily activities of the Tropidurus hispidus (Squamata: Iguania: Tropiduridae) on the Ilha do Monte Cristo, Baía de Todos-os-Santos, Bahia State, Brazil. This paper about the Tropidurus hispidus daily activities. This species is classified as a sit-and-wait foraging and the main food items are arthropods, especially ants and termites. The lack of many aspects of behaviors related the life history of lizards, consider the great importance and relevance of studies that address these aspects. The aim of this study was to describe and analyze the daily activities of individuals of the species T. hispidus of the island of Monte Cristo, Saubara, Bahia, Brazil, relating the influence of temperature on their behavior and possible preference schedule for carrying out their activities. The data collect of group was performed using the focal animal method, each individual was observed during the period of ten minutes, and every minute, the focal animal's behavior was noted. The results presented are for a total of 300 focal records, with sampling effort of 11 hours for two observers. We concluded that individuals T. hispidus observed in Monte Cristo Island are active throughout the day, since the early hours of the morning $(06: 00 \mathrm{~h})$ until the end of the afternoon $(17: 00 \mathrm{~h})$, with peaks of activity related to the times of the day in that air temperatures were highest $\left(34.9^{\circ} \mathrm{C}\right.$ and $\left.32.9^{\circ} \mathrm{C}\right)$. Its main activity is the thermoregulation, exposed to the sun, confirming its heliothermic standard. The main substrates in which individuals are associated are trunk of trees that provide shelter and have vast food resource. The results shown on the daily activity of $T$. hispidus indicate the importance of thermoregulation process for lizards as much of their activity are associated with this aspect.

Keywords: Lizards, Behavior; Budget activities; Peak activities; Influence of temperature.

Resumo. Apresenta-se, neste trabalho, informações sobre as atividades diárias do Tropidurus hispidus. A es- 
pécie é classificada como um forrageador sedentário do tipo senta-e-espera, cujos principais itens alimentares são artrópodes, com destaque para formigas e cupins. Por não se conhecer diversos aspectos dos comportamentos relacionados à história de vida dos lagartos, consideramos a grande importância e relevância de estudos que abordem estes aspectos. Assim, o objetivo deste trabalho foi descrever e analisar as atividades diárias de indivíduos da espécie Tropidurus hispidus, da Ilha do Monte Cristo, Saubara, Bahia, Brasil, relacionando a influência da temperatura no seu comportamento e possível preferência de horário para realização das suas atividades. A coleta de dados do grupo foi feita através do método animal focal, sendo que cada indivíduo foi observado durante o período de dez minutos, e, a cada minuto, o comportamento do animal focal foi anotado. Os resultados apresentados são referentes a um total de 300 registros focais de $T$. hispidus, com esforço amostral de 11 horas, por dois observadores. Com esse trabalho podemos concluir que os indivíduos de Tropidurus hispidus observados na Ilha do Monte Cristo são ativos durante todo o dia, desde as primeiras horas da manhã (06:00 h) até o final da tarde (17:00 h), com picos de atividade relacionados aos horários do dia em que as temperaturas do ar estavam mais elevadas (34,9 ㄷ e 32,9 ㄷ). Sua principal atividade é a termoregulação, exposta ao sol, corroborando seu padrão heliófilo. Não foi observado durante o estudo preferência de horário para a realização das outras atividades, tais como deslocamento, forrageamento, territorialismo ou corte. O principal substrato em que os indivíduos estiveram associados foram troncos de árvores que provêm abrigo e possuem vasto recurso alimentar. Os resultados mostrados sobre a atividade diária de T. hispidus indicam o quanto é importante o processo de termorregulação para lagartos, já que boa parte de seus registros de atividade foram associados a este aspecto.

Palavras-chave: Lagartos, Comportamento; Orçamento de atividades; Pico de atividades; Influência da temperatura.

\section{INTRODUÇÃO}

Variações comportamentais são potencialmente vantajosas para lagartos em relação à pressão evolutiva sobre caracteres filogenéticos, devido a maior plasticidade do fenótipo diante da sua condição ectotérmica. (VIDAl et al., 2010). Uma considerável parte deste controle envolve mudanças comportamentais ao longo do dia. Em termos comportamentais, a regulação de sua temperatura pode envolver o controle do período de atividade diário (HUEY \& SLATKIN, 1976;
HATANO et al., 2001) e seleção de microhabitats. Os indivíduos movem-se ativamente à procura de locais sombreados quando sua temperatura se eleva; ou se expõem ao sol e procuram superfícies quentes quando a temperatura corporal cai, a fim de otimizar seus processos fisiológicos (AVERY, 1982; RochA et al., 2000; PIANKA \& VITT, 2003). A regulação térmica depende do hábitat em que o indivíduo se encontra e sofre influência de fatores abióticos, como irradiação direta do sol, temperatura do ar e do substrato, além de fatores bióticos, tais como comportamentos 
de forrageamento, condição reprodutiva, interações sociais, deslocamento entre ambientes sombreados e ensolarados (RochA et al., 2009). A temperatura corpórea influencia, por exemplo, a locomoção (BENNETT, 1980), as taxas metabólicas (PATTERSON \& DAVIES, 1989; BeYER \& SPOTILA, 1994), a reprodução (NAVAS \& BEVIER, 2001) e o comportamento defensivo (GALDINO et al., 2006).

Os Squamata, especialmente os lagartos, são capazes de uma termorregulação muito precisa (Pough et al., 1998), ajustando a intensidade de radiação solar que estão expostos e alternando entre áreas sombreadas e ensolaradas, escolhendo microambientes com diferentes temperaturas, com isso, ajustam o seu período de atividade, e alteram a superfície corporal exposta à radiação solar e a posição em relação ao sol (HUEY et al., 1977; Pough et al., 2003). Embora poucos lagartos consigam gerar calor metabolicamente, eles podem controlar sua temperatura corporal, mantendo-a em intervalos relativamente estreitos quando ativos, enquanto as fontes de calor estiverem disponíveis (ZUG et al., 2001; PIANKA \& VITT, 2003; KIEFER et al., 2005; RochA et al., 2009). Dessa forma, grande parte da atividade diária é gasta em interações com o ambiente, uma vez que a temperatura é um dos fatores físicos mais importantes na ecologia de lagartos (RochA et al., 2009). Os períodos de atividade de lagartos heliotérmicos ocorrem em sua maior parte no início da manhã até o início da tarde (ROCHA \& VAN-SLUYS, 2007).

A territorialidade em lagartos pode estar relacionada aos recursos alimentares, a defe- sa de parceiros ou de ninhos e aos sítios de termorregulação (FERGUSON et al., 1983). Segundo STAMPS (1983), lagartos senta-e-espera (como Tropiduridae e Agamidae), os machos geralmente defendem por meio de comportamentos agressivos sua área de vida, enquanto que em espécies forrageadoras ativas (como Scincidae, Lacertidae e Teiidae), a defesa territorial é extremamente rara. CARPENTER (1977), estudando os displays agressivos de Tropidurus, afirma que o lagarto possui comportamento territorial evidente, com displays agressivos e de confronto. Os comportamentos agressivos executados pelos machos envolvem perseguições e mordidas, e são observados principalmente quando um outro macho se aproxima de uma fêmea ou simplesmente quando entra no território alheio. No entanto juvenis e fêmeas também podem apresentar sinais comportamentais para se comunicar. Em algumas espécies de anoles por exemplo, as fêmeas podem apresentar comportamentos agonísticos para defender território quando são jovens, diminuindo sua incidência à medida que chegam à fase adulta (PIANKA E VITT, 2003), no entanto estes comportamentos ainda não foram observados em lagartos do gênero Tropidurus.

Espécies de lagartos do gênero Tropidurus (Wied-Neuwied, 1824) são diurnos, abundantes e ocupam desde formações abertas até habitats florestais (FROST, 1992; HARVEY \& GUTBERLET, 2000; Frost et al., 2001). No Brasil, o gênero é amplamente distribuído, com espécies que vivem na Amazônia, Floresta Atlântica (incluindo "Restingas"), Caatinga e Cerrado (ArAú- 
Jo, 1987; Rodrigues, 1987; Rocha \& Bergallo, 1990; VITT 1993, 1995; VITT \& CALDWELL, 1993; VITT et al., 1996; RIBEIRO et al., 2008).

Tropidurus hispidus (Spix, 1825), espécie de interesse deste estudo, pertence ao grupo dos Squamata, família Tropiduridae e é a maior espécie do gênero, com comprimento rostro-cloacal entre 68 a 114mm (Kolodiuk et al., 2010). É caracterizado pela presença de uma bolsa de ácaros em cada lado do pescoço e uma em cada lado do corpo na região postumeral (RODRIGUES, 1987). Tradicionalmente, a espécie é classificada como um forrageador sedentário do tipo senta-e-espera, cujos principais itens alimentares são artrópodes, sendo que as formigas são os mais representativos na dieta (SCHOENER, 1971; ABREU et al., 2002; VAN SLUYS et al., 2004). São lagartos territorialistas, locomovem-se pouco e apresentam coloração críptica, principal recurso para escapar de predadores (ColLI \& PAIVA, 1997). É uma espécie abundante e generalista quanto ao uso do habitat, podendo ser vista sobre os mais variados tipos de substratos: rochas, tronco de árvores, troncos caídos, pilhas de lenha, cercas de fazendas, muros, paredes, no chão (RODRIGUES, 1987). Apresenta ampla tolerância ecológica, o que permite a sua ocorrência em vários domínios morfoclimáticos, sendo encontrada na floresta atlântica, nas zonas costeiras, nas áreas de transição entre caatingas e cerrados e em afloramentos rochosos na Amazônia (VANZOLINI et al., 1980; VITT, 1995; DíAZ-URIARTE, 2000; Frost et al., 2001; ABREU et al., 2002).

Considerando a grande importância e relevância de estudos comportamentais por não se conhecer quanto afetam a história de vida dos lagartos e o fato de que ainda são escassos, o objetivo deste trabalho foi descrever e analisar as atividades diárias de indivíduos da espécie $T$. hispidus, relacionando a influência da temperatura no seu comportamento e possível preferência de horário para realização das suas atividades na Ilha do Monte Cristo, Saubara, Bahia, Brasil.

\section{MATERIAL e MÉTOdos}

Esta pesquisa foi conduzida na llha do Monte Cristo $\left(12^{\circ} 50^{\prime}\right.$ S e $38^{\circ} 49^{\prime} \mathrm{W}$ ), localizada entre o estuário do Rio Paraguaçu e Baía de Todos os Santos e, pertencente ao município de Saubara, Bahia, Brasil. A ilha possui área de $1.832 .677 \mathrm{~m}^{2}$ (Figura 1), e está inserida em uma zona climática classificada como quente e úmida, no domínio morfoclimático da Mata Atlântica (RADAM BRASIL, 1974). As temperaturas mais elevadas são registradas nos meses de janeiro a março, apresentando média de $30^{\circ} \mathrm{C} \mathrm{e}$, nos meses de julho a setembro, as temperaturas variam entre $21^{\circ} \mathrm{C}$ e $22^{\circ} \mathrm{C}$. Essa região também é caracterizada por elevado índice pluviométrico (média de precipitação anual próxima a 2.100 mm/ano), com chuvas bem distribuídas durante o ano (RADAM BRASIL, 1974).

As observações em campo foram realizadas nos dias 14 e 15 de julho de 2015, ao longo de três pontos amostrais escolhidos na llha, distantes aproximadamente 100 metros uns dos outros, denominados de "casa", "depósito" e "ruína". Duas pessoas permaneceram pelo menos 


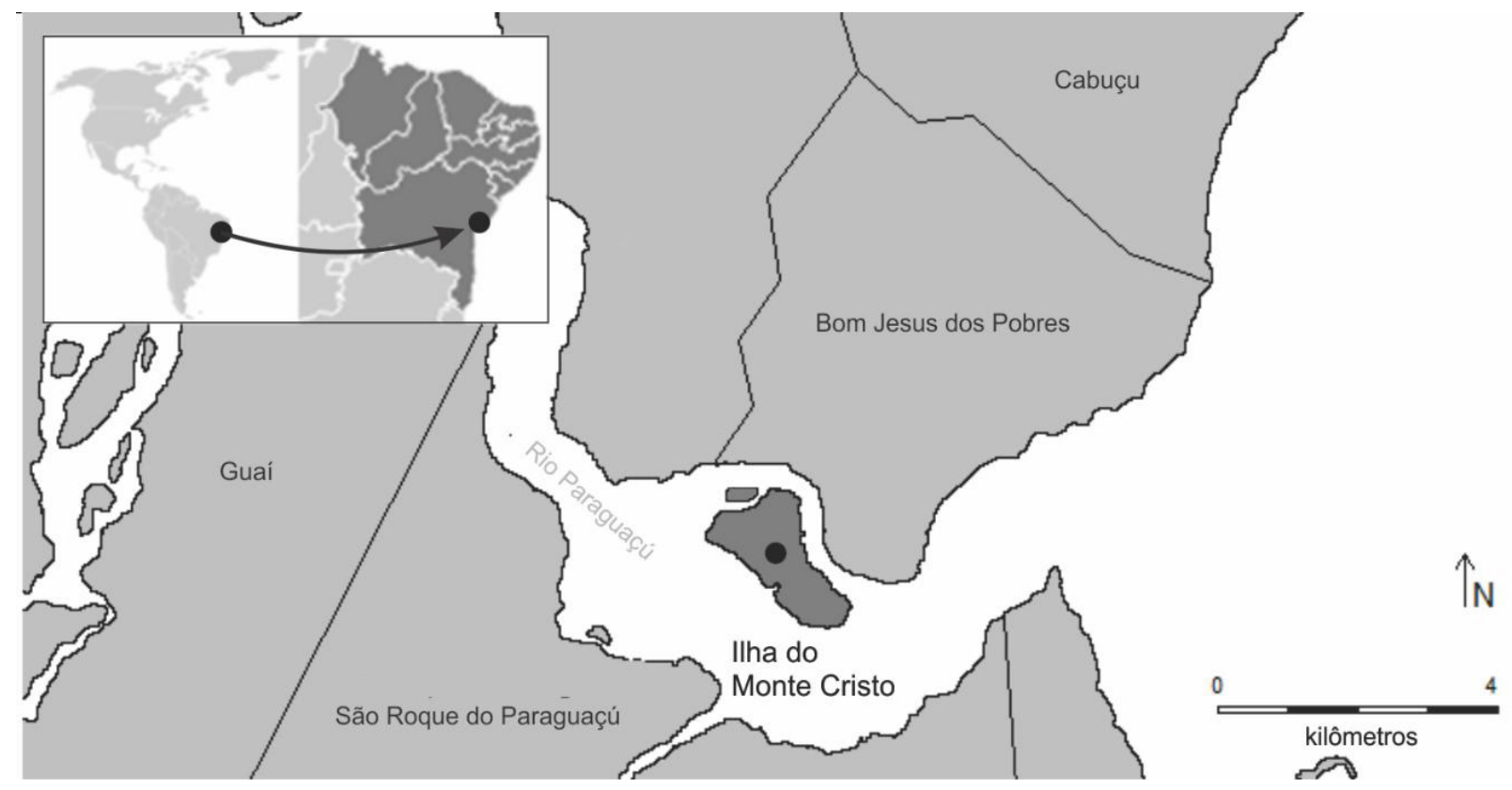

Figura 1. Mapa da localização da ilha do Monte Cristo, Saubara, Bahia, Brasil.

uma hora em cada ponto amostral, realizando busca ativa, das 06h:00min às 17h:00min. Para cada lagarto foram registradas as temperaturas do ar e do substrato por meio de um relógio termo-higrômetro no local onde cada indivíduo foi localizado pela primeira vez.

A coleta de dados de comportamento foi feita através do método animal focal (ALTMANN, 1974), que consiste em observar os animais por um determinado tempo e a cada minuto, anotar o comportamento que o animal está executando. Neste estudo, cada indivíduo foi observado durante o período de dez minutos, e, a cada minuto, o comportamento do animal focal era anotado, com a finalidade de elaborar um quadro das atividades diárias realizadas pela espécie. Foram descartados os focais em que os espécimes observados permaneceram fora de visibilidade por mais de dois minutos.
Além das atividades diárias, foram registradas as seguintes informações para cada lagarto observado: data; hora do dia; exposição do lagarto à luz solar (totalmente exposto ao sol, à sombra ou a um mosaico de sol e sombra); e o substrato em que o indivíduo esteve durante os dez minutos de observação. Todas essas informações foram registradas por meio de um gravador portátil (V-SENSOR TP-VS480 AIWA).

\section{Resultados e Discussão}

Os resultados apresentados são referentes a um total de 300 registros focais de 30 indivíduos de T. hispidus, com esforço amostral de 11 horas. Ao longo deste tempo foi possível registrar as atividades diárias de: imóvel, deslocamento, forrageamento, comportamento agonístico e outros (Tabela 1).

Foi registrado um comportamento de 
Tabela 1. Atividades registradas durante a amostragem de Tropidurus hispidus, na Ilha do Monte Cristo, Saubara, Bahia, Brasil.

Atividades

Imóvel

Forrageando

Comportamento agonístico

Deslocando

Outros

\section{Descrição}

Quando o lagarto se encontra parado apresentando apenas alterações no grau de achatamento do corpo para aumentar ou reduzir a superfície de contato em relação ao substrato e a radiação solar direta.

Quando o lagarto se movimenta dentro do micro-habitat e é observado predando ou em tentativa de predação.

Quando o lagarto apresenta comportamento agressivo para afastar outro lagarto (da mesma espécie ou não).

Quando o indivíduo se desloca dentro do micro-habitat para qualquer outra atividade que não esteja ligada à sua alimentação.

Comportamentos exibidos que não se encaixam nas definições previas do estudo (corte, cópula). corte durante a amostragem (incluído na categoria outros, $n=1 ; 0,3 \%)$, onde o macho ficou bem próximo da fêmea, dando voltas ao redor da mesma, fazendo movimentos rítmicos com a cabeça e corpo para cima e para baixo, mostrando a região gular distendida. A fêmea ao aceitar a corte, tomou a posição de costas para o macho, com o dorso levemente curvado e a cauda apontada para cima, pronta para a cópula efetiva. Porém, o macho não realizou a cópula com a introdução do hemipênis.

Observou-se que os indivíduos de T. hispidus passaram a maior parte do seu tempo imóvel $(n=229 ; 76,3 \%)$, seguido de diversas outras atividades (Figura 2). O registro da imobilidade na maior parte do tempo, se deve porque este é um comportamento típico de lagartos senta-espera, que é uma das estratégias utilizadas para a captura de presas móveis que vão ao seu encontro (RochA, 1994). No entanto este tempo imóvel pode também ser associado ao processo de termorregulação, pois é comum que os répteis passem a maior parte do tempo expostos ao sol se aquecendo, comportamento conhecido como "heat-gain" (VITT \& CALDWELL, 2013). A capacidade dos ectotérmicos em manter a temperatura corporal dentro de um estreito intervalo é possível graças aos mecanismos de termoregulação.

Com relação aos horários de atividade, observou-se animais ativos durante todo o período (6h:00min às $17 \mathrm{~h}: 00 \mathrm{~min}$ ), com padrão bimodal, com dois picos de atividades ocorrendo das 08h:00min às 08h:59min e das 13h:00min às 13h:59min (Figura 3). O pico de atividades nesses horários esteve relacionado à temperatura do ar, visto que esses foram os horários com as maiores temperaturas do dia (Figura 4), favorecendo a termorregulação, concordando com outros estudos para os lagartos do gênero Tropidurus que discutem que este padrão de atividade diária está relacionado com a variação da temperatu- 


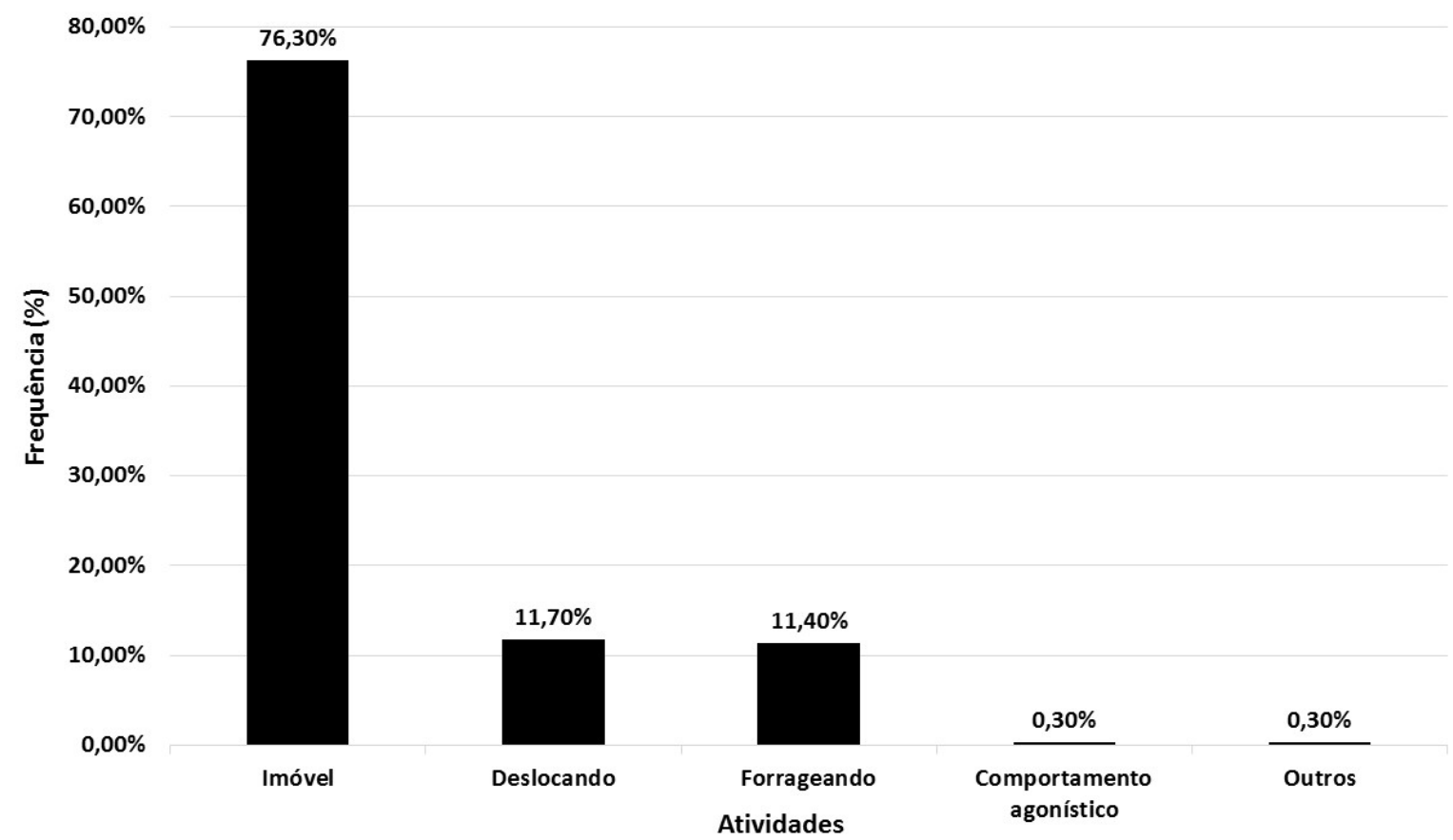

Figura 2. Atividades diárias desenvolvidas pelos lagartos Tropidurus hispidus da Ilha do Monte Cristo, Saubara, Bahia, Brasil, durante o período de observação.

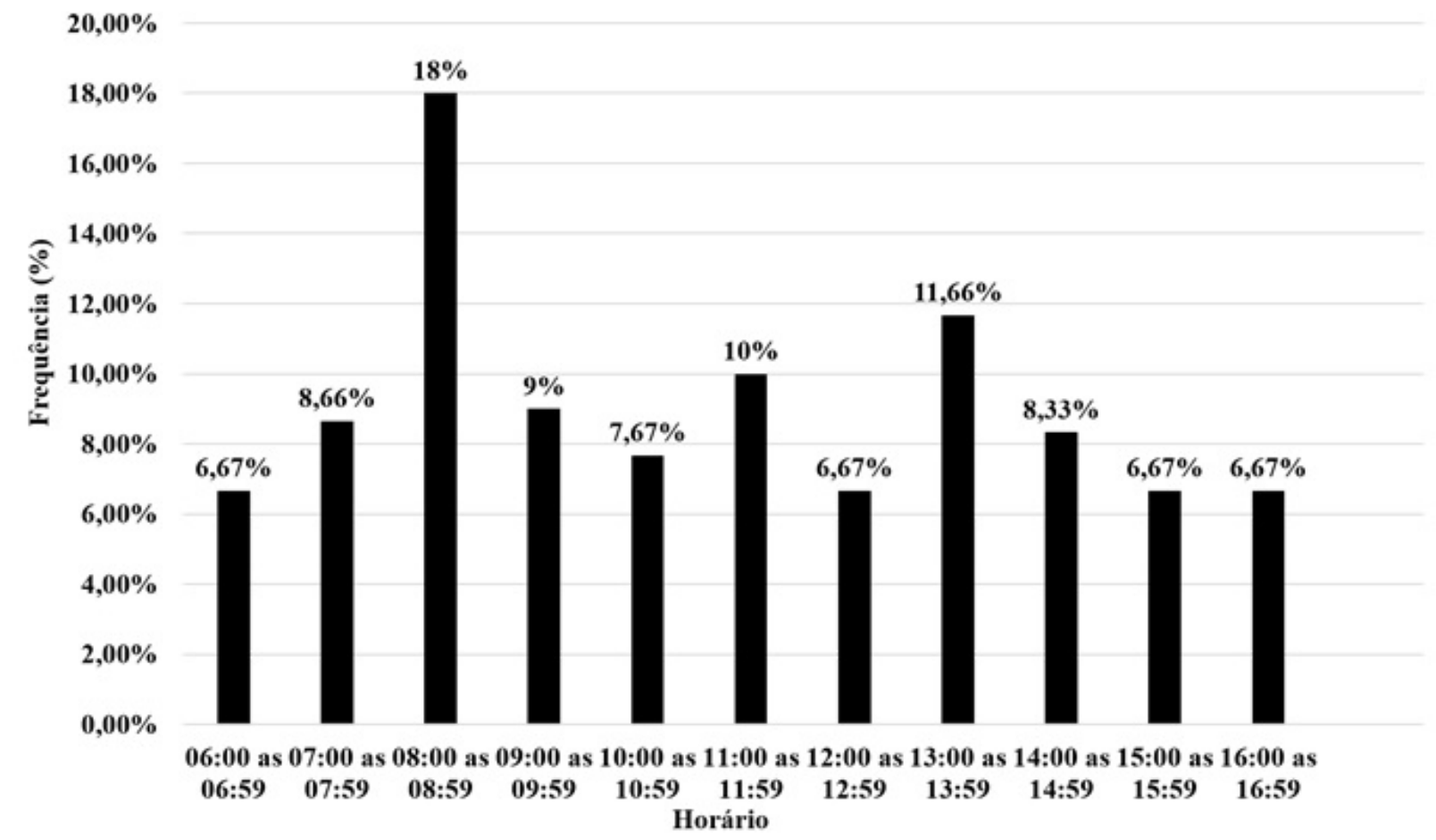

Figura 3. Horários de atividade dos lagartos Tropidurus hispidus da Ilha do Monte Cristo, Saubara, Bahia, Brasil. 


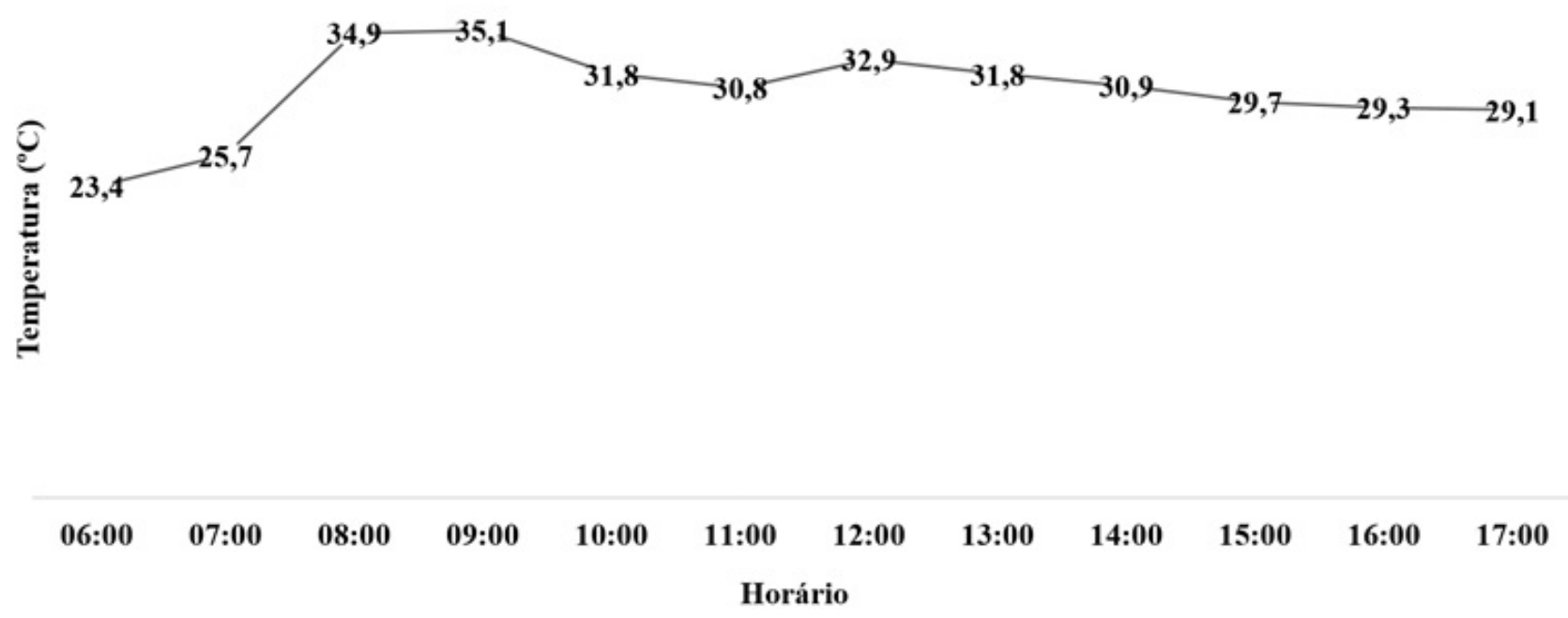

Figura 4. Temperatura do ar durante o período de observação dos lagartos Tropidurus hispidus da Ilha do Monte Cristo, Saubara, Bahia, Brasil.

ra do ambiente ao longo do dia, o que favorece aos indivíduos regular sua temperatura e realizar a maioria dos seus comportamentos (VITT, 1983, 1995; Rocha \& BERGALLO, 1990;VAN SLUYS, 1992; VITT, 1993; CRUz et al., 1998; HATANO et al., 2001; RIBEIRo et al., 2009; RochA et al., 2009).

Indivíduos de T. hispidus foram observados principalmente expostos totalmente ao sol ( $n=128 ; 42,6 \%$ ) (Figura 5 ), comportamento comum em lagartos heliófilos, de áreas abertas, que necessitam de uma maior exposição à luz durante a termorregulação (VITT, 1983; RocHA \& BERGALLO, 1990). Em estudo semelhante com a mesma espécie, em ambiente continental, os animais também apresentaram hábitos de termorregulação e deslocamento com maior frequência durante as observações (VITT et al. 1996). Esse trabalho, também encontrou correlações entre os períodos mais quentes e os períodos de maior atividade na espécie sugerindo forte influência da temperatura ambiental nas atividades desenvolvidas pela espécie.

O principal substrato utilizado pelos Tropidurus da Ilha do Monte Cristo foi troncos de árvores ( $n=60 ; 60,6 \%$ ) (Figura 6). Segundo GROVER (1996), lagartos que utilizam habitats próximos de árvores recebem mais sombra ao meio dia, quando as temperaturas são mais extremas e, consequentemente, microhabitats próximos às árvores podem permitir ao animal ter maior área de vida e período de atividade. $\mathrm{O}$ grande número de registros de indivíduos encontrados em árvores pode ser explicado inclusive pelo fato de nesses locais, serem encontrados diversos pontos de incidência solar durante o dia, assim, há uma maior variação de temperatura e os lagartos podem termorregular com maior eficiência, apenas deslocando-se na árvore, que também oferecem maior disponibilidade de alimento, tais como formigas e cupins. 


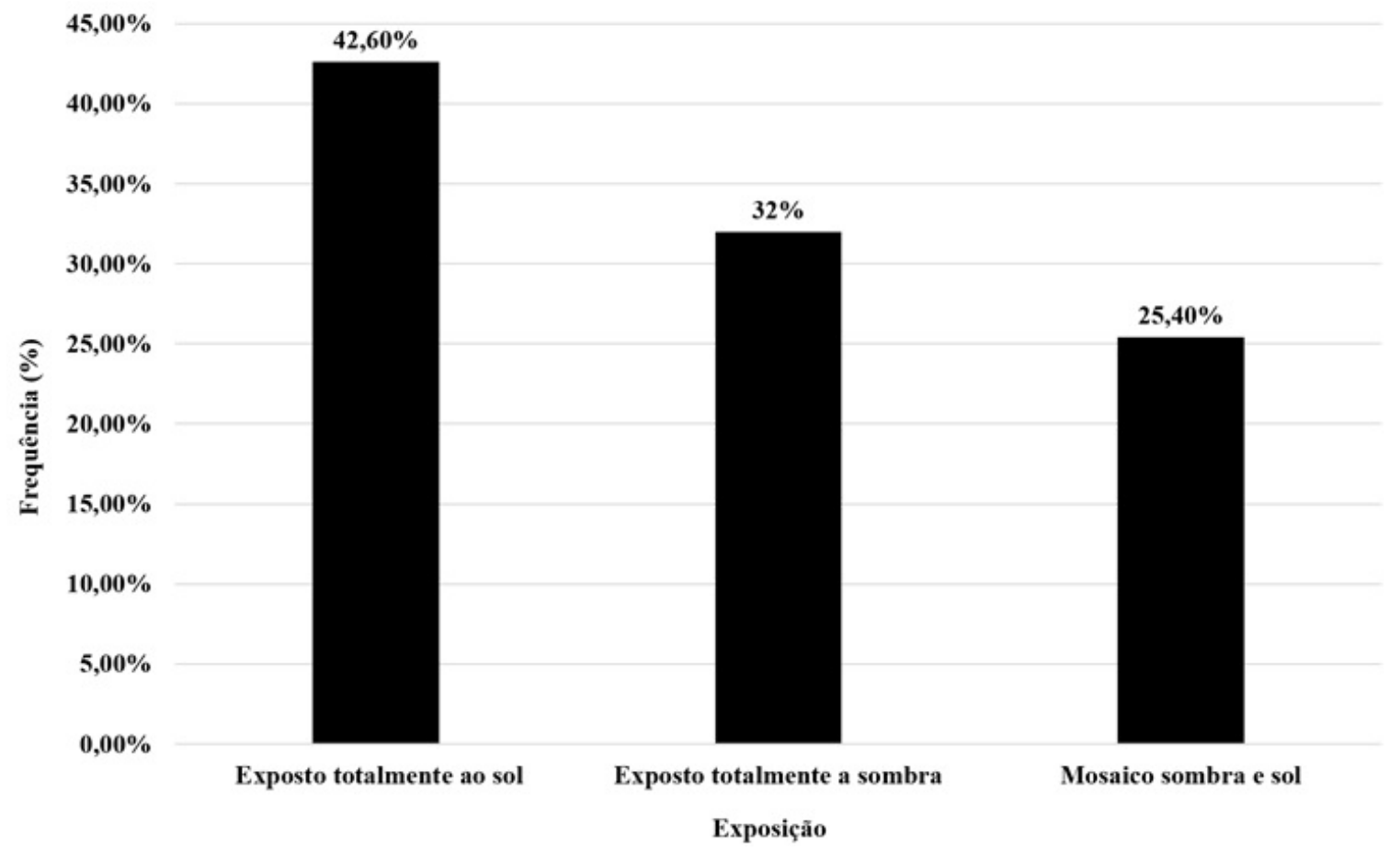

Figura 5. Frequencia de exposição dos lagartos Tropidurus hispidus à luz, Ilha do Monte Cristo, Saubara, Bahia, Brasil, durante o período de observação.

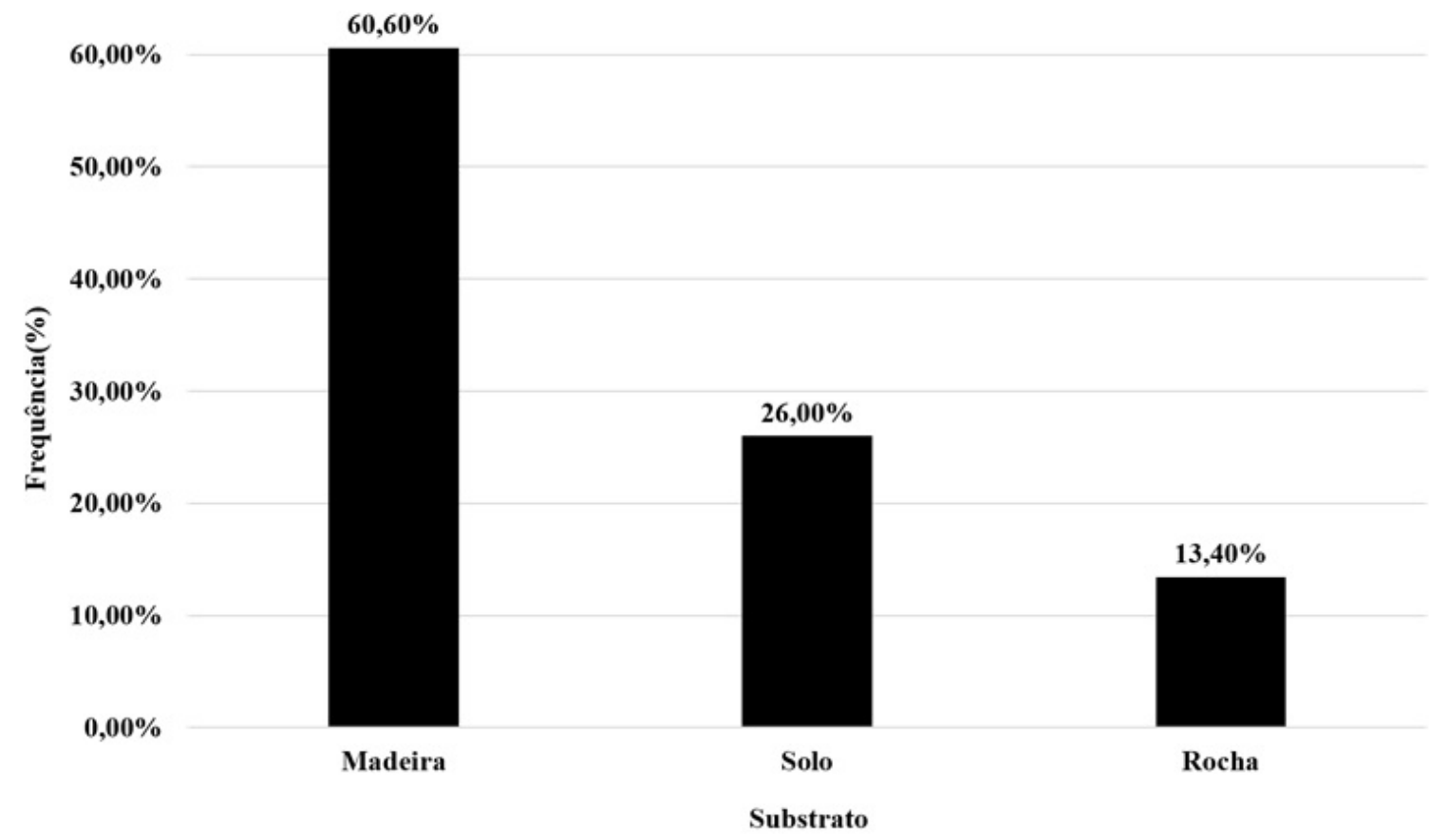

Figura 6. Uso do substrato pelos lagartos Tropidurus hispidus da Ilha do Monte Cristo, Saubara, Bahia, Brasil. Período de observação (06:00 h às 18:00 h). 
Ambientes com flutuações térmicas ao longo do dia favorecem a presença de lagartos com comportamento senta-espera. Nestes locais os lagartos podem se expor as temperaturas mais elevadas no início do dia quando começam sua atividade diária e à medida que se tornam mais aquecidos podem buscar as porções do habitat com temperaturas mais amenas a fim de evitar superaquecimento (e. g. RochA, 1995; RIBEIRO et al. 2009). Os troncos de árvores no nosso estudo mostraram-se uteis neste aspecto para $T$. hispidus. No entanto, T. hispidus apresenta uma grande plasticidade de nicho espacial utilizando uma ampla variedade de substratos, sendo encontrados em superfícies rochosas (VITT, et al., 1997; VAN-SLYUS, et al., 2004), bordas de mata (Rodrigues, 1987; CARVAlHo, et al., 2005), troncos de árvores, solos arenosos, cercas e muros de construções humanas, entre outros (RODRIGUES, et al., 1987). Essa variedade de ambientes explorados faz dessa espécie um excelente modelo de estudo sobre plasticidade ecológica. Nossos resultados reforçam os outros trabalhos, mostrando a condição generalista da espécie em relação a ocupação de microhabitats que pode ser dependente dos recursos disponíveis em abundância no ambiente.

\section{CONCLUSÃo}

Conclui-se que os indivíduos de Tropidurus hispidus observados na Ilha do Monte Cristo, estiveram ativos durante todo o dia, desde as primeiras horas da manhã até o final da tarde, com picos de atividade bimodais afetados pela ação da temperatura do ambiente. Os indivíduos passam a maior parte do tempo imóvel, expondo-se ao sol, de acordo como seu padrão heliófilo. Não há uma preferência de horário para a realização das outras atividades, tais como deslocamento, forrageamento e comportamentos agonísticos. O principal substrato em que os indivíduos estão associados são troncos de árvores que provêm abrigo, possibilidade de rápido deslocamento e alimentação. Os resultados mostrados sobre a atividade diária de T. hispidus indicam o quanto é importante o processo de termorregulação para lagartos, já que boa parte de seus registros de atividade foram associados a este aspecto. Devido a esta condição acreditamos que os outros comportamentos devam aparecer ao longo do dia e variem à medida que o animal alcance temperaturas ideais para desenvolve-los.

\section{AgradeCIMENTOS}

Os autores agradecem a Ana Ruth Rodrigues de Jesus pelo auxílio na coleta de dados, ao PPGDA/UFBA - Programa de Pós-graduação em Diversidade Animal da Universidade Federal da Bahia pelo auxílio financeiro, uma vez que esta pesquisa foi conduzida no âmbito das disciplinas "Zoologia de Campo I" e "História Natural de Squamata" e a FAPESB- Fundação de Amparo à pesquisa do estado da Bahia pelo apoio financeiro.

\section{REFERÊNCIAS BIBLIOGRÁFICAS}


Abreu M.L. De S.; Frota J.G. \& YUKI R.N. 2002. Geographic distribution of Tropidurus hispidus. Review Herpetology 33: 66.

AltmanN, J. 1974. Observational study of behaviour: sampling methods. Behaviour 49: 227-267.

ARAújo, A.F.B. 1987. Comportamento alimentar dos lagartos: O caso dos Tropidurus da Serra dos Carajás, Pará (Sauria: Iguanidae). In: Encontro Anual de Etologia. Anais de Etologia, Ribeirão Preto, Jaboticabal: Funep, 5: 203-234.

AVERY, R.A. 1982. The paper of termorregulation in the biology of the lizard: predatory in basker diurnal temperate. Behavioral Ecology and Sociobiology 11: 261-267.

BENNETT, A.F., 1980. The thermal dependence of lizard behaviour. Animal Behavioral. 28 (3), 752-762.

BEYER, E.C. \& SPOTILA, J.R., 1994. Seasonal variation in metabolic rates and maintenance costs of the eastern fence lizard, Sceloporus undulatus. Comparative Biochemistry Physiology A-Comp. Physiol. 109 (4), 1039-1047.

CARPENTER, C. C. 1977 . The agressive displays of three species of South American Iguanid lizards of the Genus Tropidurus. Herpetologica, 33: 285-289.

Carvalho, C.M.; Vilar, J.C.; \& Oliveira, F.F. 2005.
Répteis e anfíbios. In Carvalho, C. M.; Vilar, J. C. (coord.). Parque Nacional Serra de Itabaiana - Levantamento da Biota. Aracaju: IBAMA, p. 39-61.

ColLI, G.R. \& PAIVA, M.S. 1997. Estratégias de forrageamento e termorregulação em lagartos do Cerrado e Savanas Amazônicas, pp. 224- 231. In: Leite, L.L. \& Saito, C.H. (orgs.). Contribuição ao conhecimento ecológico do Cerrado. Editora da Universidade de Brasília, Brasília. 224-231.

CRUZ, F.B.; SILVA, S. \& SCROCCHI, G.J. 1998. Ecology of the lizard Tropidurus etheridgei (Squamata: Tropiduridae) from the dry chaco of Salta, Argentina. Herpetological Natural History 6 (1): 23-31.

DÍAZ-URIARTE, R. 2000. Effects of aggressive interactions on antipredator behavior: empirical and theoretical aspects. Tese de Doutorado. University of Wisconsin, Madison. $145 p$.

Ferguson, G. W.; HugheS, J. L.; \& BroWN, K. L. 1983. Food availability and territorial establishment of juvenile Sceloporus undulatus. In: Huey, R. B.; Pianka, E. R. e Schoener, T. W. Lizard Ecology. Harvard University Press, Cambridge. 501 p.

Frost, D.R. 1992. Phylogenetic analysis and taxonomy of the Tropidurus Group of Lizards (Iguania: Tropiduridae). American Museum 3033: 1-68. 
FROST, D.R.; RODRIGUES, M.T.; GRANT, T. \& TITUS, T.A. 2001. Phylogenetics of lizards genus Tropidurus (Squamata: Tropiduridae: Tropidurinae): Direct optimization, descriptive efficiency, and sensivity analysis of congruence between molecular data and morphology. Molecular Phylogenetics and Evolution 21 (3): 352-371.

Galdino, C. A. B.; Pereira, E. G.; Fontes, A. F.; \& VAN SLUYS, M. 2006. Defense behavior and tail loss in the endemic lizard Eurolophosauros nanuzae (squamata, Tropiduridae) from southeastern Brazil. Phyllomedusa, 5(1): $25-30$.

GROVER, M.C. 1996. Microhabitat use and thermal ecology of two narrowly sympatric Sceloporus (Phrynosomatidae) lizards. Journal of Herpetology 30 (2): 152-160.

HaRVey, M.B. \& Gutberlet, R.L. 2000. A phylogenetic analysis of the tropidurine lizards (Squamata: Tropiduridae), including new characters of squamation and epidermal microstructure. Zoological Journal of the Linnean Society 128: 189-233.

Hatano, F.H.; VRcibradic, D.; Galdino, C.A.B.; CUNHA BARROS, M.; ROCHA, C.F.D. \& VAN SLUYS, M. 2001. Thermal ecology and activity patterns of the lizard community of the Restinga of Jurubatiba, Macaé, RJ. Revista Brasileira de Biologia 61 (2): 287-294.

HUEY, R.B. \& SLATKIN, M. 1976. Costs and benefits of lizard thermoregulation. Quarterly Review of Biology 51: 363-384.

HUeY, R. B.; PIANKA, E. R.; \& Hoffman, J. A. 1977. Seasonal variation in thermoregulatory behavior and body temperature of diurnal Kalahari lizards. Ecology, 58(5): 1066-1075.

KiEfER, M.C.; VAN-SLUYS, M. \& RochA, C.F.D. 2005. Body temperatures of Tropidurus torquatus (Squamata, Tropiduridae) from coastal populations: Do body temperatures vary along their geographic range? Journal of Thermal Biology 30: 449-456.

Kolodiuk, M. F.; RIBEIRO, L. B. \& FreIRE, E.M.X. 2010. Diet and Foraging Behavior of Two Species of Tropidurus (Squamata, Tropiduridae) in the Caatinga of Northeastern Brazil. South American Journal of Herpetology 5 (1): 35-44.

NAVAS, C.A. \& BeVIER, C. 2001. Thermal dependency of calling performance in the eurythermic frog Colostethus subpunctatus. Herpetologica 57, 384-395.

Patterson, J.W. \& DaVies, P.M.C., 1989. A possible effect of climate on resting metabolic rate in lizards. Copeia, 719-723.

PIANKA, E. R. \& VITT, L. J. 2003. Lizards: windows to the evolution of diversity. London, University of California Press. 333p.

Pough, H.F. ANDREWS, R.M., CADle, J.E., CRumP, M.L., SAVITZKY, A.H. \& WellS, K.D. 1998. 
Herpetology. Prentice- Hall, New Jersey, USA.

Pough, F. H.; JANIS, C. M.; \& HeISER, J. B. 2003. A vida dos vertebrados. São Paulo: Atheneu. $839 \mathrm{p}$.

RADAM BRASIL. 1974. Folha SA 22. Belém; geologia, geomorfologia, solos, vegetação e uso potencial da terra. Rio de Janeiro: DNP/ MME. Projeto RADAM BRASIL. Vol. 5, 478 pp.

RIBEIRO, L.B.; GoMIDES, S. C.; SANTOS, A.O. \& SOUSA. B. M. 2008. Thermoregulatory behavior of the saxicolous lizard, Tropidurus torquatus (squamata, tropiduridae), in a rocky outcrop in Minas Gerais, Brazil. Herpetological Conservation and Biology 3(1):6370.

Ribeiro, L. B.; SOUSA, B. M.; \& Gomides, S. C. 2009 Range structure, microhabitat use, and activity patterns of the saxicolous lizard Tropidurus torquatus (Tropiduridae) on a rock outcrop in Minas Gerais, Brazil. Revista Chilena de História Natural. 82: 577588.

RochA, C.F.D. \& Bergallo, H.G. 1990. Thermal Biology and Flight Distance of Tropidurus oreadicus (Sauria, Iguanidae) in an area of Amazonian Brazil. Ethology Ecology and Evolution 2: 263-268.

RocHA, C.F.D. 1994. Introdução à ecologia de la- gartos brasileiros, p.39-37.. In: Nascimento L.B.; Bernardes A.T. \& Cotta, G.A. (eds). Herpetologia no Brasil I. PUC/MG, Fundação biodiversitas \& Fundação Ezequiel Dias, Minas Gerais. 39-57.

RocHA, C.F.D. 1995. Ecologia termal de Liolaemus lutzae (Sauria: Tropiduridae) em uma área de restinga do sudeste do Brasil. Revista Brasileira de Biologia 55:481-489.

Rocha, C.F.D.: VRciBradic, D. \& ARaújo, A.F.B. 2000. Ecofisiologia de répteis de restinga brasileiras, p. 117-149. In: Esteves, F.A. \& Lacerda, M. (eds.) Ecologia de Restingas e Lagoas Costeiras. NUPEM/UFRJ, Macaé. 117-149.

ROCHA, C.F.D. \& VAN SLUYS, M. 2007. Herpetofauna de restingas, p. 44-65. In: Nascimento, L. B. \& Oliveira, M. E. (orgs.). Herpetologia no Brasil II. Sociedade Brasileira de Herpetologia, Belo Horizonte, .44-65.

ROCHA, C.F.D. \& VAN SLUYS, M.; VRCIBRADIC, D.; Kiefer, M.C.; Menezes, V.A.; SIQUeIRA, C.C. 2009. Comportamento de termorregulação de lagartos brasileiros. Oecologia Brasiliensis 13 (1): 115-131.

RODRIGUES, M.T. 1987. Sistemática, Ecologia e Zoogeografia dos Tropidurus do Grupo Torquatus ao Sul do Rio Amazonas (Sauridae, Iguanidae). Arquivos de Zoologia de São Paulo 31: 105-230. 
SCHOENER, T.W. 1971. Theory of feeding strategies. Annual Review of Ecology and Systematics 2: 369-404.

StAMPS, J. A. 1983. Sexual Selection, Sexual Dimorphism, and Territoriality. In: Huey, R. B.; Pianka, E. R. e Schoener, T. W. Lizard Ecology. Harvard University press, Cambridge. 501 p.

VAN SLUYS, M. 1992. Aspectos da ecologia do lagarto Tropidurus itambere (Iguanidae) em uma área do sudoeste do Brasil. Revista Brasileira de Biologia 52: 181-185.

VAN-SlUYS, M.; RochA, C.F.D.; VRCIBRADIC, D.; GALDINO, C.A.B. \& FonTES, A.F. 2004. Diet, activity, and Microhabitat use of two syntopic Tropidurus species (Lacertilia: Tropiduridae) in Minas Gerais, Brazil. Journal of Herpetology 38(4): 606-611.

VANZOLINI, P.E.; CoSTA-RAMOS, A.M.M. \& VITT, L.J. 1980. Répteis da Caatinga. Rio de Janeiro: Academia Brasileira de Ciências. 161p.

VIDAL, M.A.; HABIT, E.; Victorino, P.; GonZÁleZ-Gajardo, A.; \& ORTIZ, J.C. 2010. Thermoregulation and activity pattern of the high-montain lizard Phymaturus palluma (Tropiduridae) in Chile. Zoologia 27(1): 13 $-18$.

VITT, L.J. 1983. Tail loss in lizards: The significance of foraging and predation escape modes. Herpetologica 39(2):151-162.
VITT, L.J. 1993. Ecology of isolated open-formation Tropidurus (Reptilia: Tropiduridae) in Amazonian lowland rain forest. Canadian Journal of Zoology 71: 2370-2390.

VITT, L.J. \& CALDWELL, J.P. 1993. Ecological observations on Cerrado Lizards in Rondônia, Brazil. Journal of Herpetology 27 (1): 4652.

VITT, L.J. 1995. The ecology of tropical lizards in the caatinga of Northeast Brazil. Occasional Papers of the Oklahoma Museum of Natural History 1: 1-29.

VITT, L.J.; ZANNI, P.A. \& CALDWELL, J.P. 1996. Behavioural ecology of Tropidurus hispidus on isolated rock outcrops in Amazonia. Journal of Tropical Ecology, 12: 81-101.

VITT, L. J., AVILA-PIRES, T. C. S. \& ZANI, P. A. 1996. Observations on the ecology of the rare amazonian lizards, Enyalius leechii (Polychrotidae). Herpetological Natural History. v. 4, n.1, p. 77-82.

VITT, L.J.; CALDWELL, J.P.; ZANI, P.A.; \& TITUS, T.A. 1997. The role of habitat shift in the evolution of lizard morphology: evidence from tropical Tropidurus. Proceedings of the National Academy of Sciences. U.S.A., 94: 38283832.

VITT, L. J., \& CALDWELL, J. P. 2013. Herpetology: an introductory biology of amphibians and reptiles. Academic Press. 
ZUG, G.R.; VITT, L.J. \& CALDWELL, J.P. 2001. HerpeRecebido: 18/02/2016 tology: An Introductory Biology of AnphibRevisado: 04/09/2016 ians and Reptiles. 2 ed. Academic press, Aceito: 05/10/2016 San Diego; California. 
70. LIMA et al.

Revista Brasileira de Zoociências 18(2): 55-70. 2017 